\title{
Sur les essais de prévision des apports de Printemps et d'Été 1949 dans certains réservoirs
}

\section{Tests for estimating the apports of Spring and Summer 1949 in certain reservoirs}

\author{
PAR M. S. FERRY \\ Cher de la Division techngue générale du Service de la Production hydrauloue \\ Dmection de l'Pxploitation, Electricté de Francle
}

English synopsis p. 193

Dans une communication précédente au Comité Technique (1), j’ai indiqué les grandes lignes des méthodes employées par le service de la Production hydraulique d'Electricité de France dans le but d'évaluer l'enneigement dans les bassins de montagne.

J'ai donné aussi quelques indications sur l'utilisation des matériaux d'observation, pour la prévision des apports globaux de la période de fonte des neiges. Cette prévision étant essentiellement basée sur des méthodes statistiques nécessite done des observations aussi étendues que possible dans le passé. Or, les seules données actuellement disponibles sur des séries de quelque importance touchent les précipitations, la température et, naturellement, l'écoulement. Ce sont donc ces éléments que nous devons utiliser actuellement pour la prévision; les mesures directes de l'enneigement ne servent pour l'instant que de recoupement et sont surtout destinées à constituer des matériaux statistiques nouveaux pour l'avenir. Nous envisageons aussi d'utiliser ultérieurement d'autres observations telles que l'insolation, l'évaporation ou l'humidité de l'air, lorsqu'on aura pu en constituer des séries suffisantes.

L'objet de la présente communication est de faire connaître au Comité Technique quelques exemples concrets d'application pratique, tels qu'ils se sont effectivement présentés cette année 1949, caractérisée par une insuffisance exception-

(1) Voir la Houille Blanche, numéro spécial «A»1949, pp. 360 et 361. Voir aussi « les Méthodes d'estimation de l'emneigement dans les bassins de montagne et leur utilisation à la prevision des apports de printemps et d'été $\gg$, rapport de M. S. Ferry au Congrès de Bruxelles (septembre 1949) de l'Union internationale des Producteurs et Distributears d'Energic, 12, place des Etats-Unis, Paris (XVI"). nelle des précipitations tant pendant l'hiver 194849 que pendant la période de fonte des neiges.

Avant d'aller plus loin, je voudrais insister quelque peu sur la nature et le but de nos prévisions. Ces dernières concernent, je le répète, les apports globaux de quelques bassins de montagne pendant des périodes de quelques mois influencées par la fonte des neiges. J'emploie le mot «prévisions» à défaut d'un autre plus approprié et je précise tout de suite qu'il ne s'agit pas de « prédiction ». Nous opérons, en effet, dans un domaine où il ne saurait être question d'annoncer avec certitude la réalisation d'apports bien déterminés, avec une marge d'erreur assez réduite pour être pratiquement négligeable. Des possibilités d'écarts importants subsisteront toujours, ne serait-ce qu'en raison des précipitations à intervenir pendant la période faisant l'objet de la prévision.

Cette dernière consiste donc à indiquer la probabilité attribuable à la réalisation d'écoulements supérieurs (ou inférieurs) à toute valeur donnée, et cela, compte tenu des éléments susceptibles d'influencer cette distribution de probabilités et connus au moment de la prévision.

Nous cherchons ainsi à donner la meilleure mesure possible des risques courus en adoptant telle ou telle politique d'exploitation.

Le principe même de la méthode est bien connu; il consiste à rechercher le lien de corrélation, généralement supposé linéaire, entre la quantité à prévoir et un certain nombre de grandeur's influençant celle-ci. M. Coutagne, notamment, a fait ici de nombreuses communications utilisant cette notion de corrélation et montré tout l'intérêt qu'elle présente.

Pratiquement, le calcul est conduit de la façon suivante : 
$1^{\circ}$ Etude de la distribution de l'écoulement de la période envisagée, indépendamment des caractéristiques de l'hiver.

Nous avons admis dans la plupart des cas qu'une distribution de Gauss pouvait être ajustée sur le graphique de fréquence des écoulements de la période de référence et que, par suite, la distribution pouvait être définie par la moyenne des valeurs constatées pendant cette période, soit $\mathrm{E}_{0}$, et par l'écart-type $\sigma_{\mathrm{L}}$.

$2^{\circ}$ Choix des éléments météorologiques connus à la date de la prévision et présentant un lien de corrélation suffisamment étroit avec l'écoulement de la période à venir.

Jusqu'à présent, nous avons utilisé, soit les précipitations totales de la période d'hiver, soit la partie de ces précipitations accumulées sous forme de réserves solides ou liquides dans le bassin et subsistant à la date de la prévision, représentée par une quantité de la forme :

$$
k \mathrm{P}_{\text {II }}-\mathrm{E}_{\mathrm{II}}
$$

$P_{11}=$ moyenne des précipitations recueillies dans un certain nombre de stations pendant la saison d'hiver;

$\mathbf{E}_{\mathrm{II}}=$ écoulement de la période d'hiver;

soit enfin, dans quelques cas, l'écoulement du ou des mois précédents, celui-ci reflétant en partie l'état des réserves liquides du bassin.

Nous appellerons. H l'élément météorologique ou la combinaison d'éléments météorologiques retenue pour la corrélation.

$3^{\circ}$ Calcul des éléments de corrélation, admise linéaire, c'est-à-dire essentiellement :

Coefficient de corrélation $r$

et Coefficient de régression $b$

(régression $\mathrm{E} / \mathrm{H}$ )

$4^{\circ}$ Détermination de la nouvelle valeur la plus probable de l'écoulement $\mathrm{E}$ au moyen de l'équation de régression :

$$
\overline{\mathrm{E}}=\mathrm{E}_{0}+b\left(\mathrm{H}-\mathrm{H}_{0}\right)
$$

( $\overline{\mathbf{E}}$ symbolisant l'espérance mathématique de $\mathbf{E}$ pour une valeur donnée de $\mathrm{H}$ ).

$5^{\circ}$ Détermination de la nouvelle répartition en fréquence des diverses valeurs d'écoulement possibles.

Cette répartition est donnée par une distribution de Gauss dont la moyenne est la valeur $\overline{\mathrm{E}}$ et dont l'écart-type $\sigma_{\mathrm{E}}^{\prime}$ est égal à l'écart-type initial $\sigma_{\mathrm{x}}$ multiplié par un coefficient plus petit que 1 et égal à $\sqrt{ } \mathbf{1}^{-r^{2}}$.

$$
\sigma_{\mathrm{E}}^{\prime}=\sigma_{\mathrm{I}} \times \sqrt{1-r^{2}}
$$

Ayant en mains les éléments de la prévision, celle-ci est énoncée en faisant ressortir les va- leurs des apports qui onl 95, 90,50 chances sur 100 d'être dépassées.

On en déduit ensuite les taux de probabilités à escompter pour le remplissage total ou partiel de la réserve ou pour les disponibilités excédant la capacité de la réserve.

Quelques exemples de prévisions effectivement énoncées cette année et relatives à des bassins alpestres vont éclairer davantage mon exposé.

\section{Drac au Sautet.}

Le Drac au Sautet est le premier bassin que nous avons étudié car nous disposions d'une série de renseignements sur les précipitations et les écoulements remontant à 1927, l'ex-Société Bonne et Drac s'étant intéressée à la question et ayant installé un réseau assez dense de stations pluviométriques depuis cette date.

Le tableau I indique les périodes ayant fait l'objet de prévisions en 1949 et l'élément hydrologique $\mathrm{H}$ retenu pour le calcul de corrélation.

TAmLeaU I

PREVISION DES APPORTS DE PRINTEMPS ET D'ÉTÉ 1949

Drac aut Salutet

Périodes ayant fait lobjet de prévisions

\begin{tabular}{|c|c|}
\hline PÉRIONE & ELEMENT $H$ \\
\hline 1 or mars-31 aoul : & $\left\{\begin{array}{l}\mathrm{P}_{\mathrm{H}}=\text { précipitations totales } \\
\text { représentées par la moyen- } \\
\text { ne arithméticue des préci- } \\
\text { pitations recueillies dans } \\
12 \text { stations pendant la pé- } \\
\text { riode } 1^{\text {er }} \text { novembre-28 fé- } \\
\text { vrier. }\end{array}\right.$ \\
\hline 1er avril-31 aout : & $\left\{\begin{array}{l}\mathrm{P}_{\mathrm{II}}=\text { précipitations totales } \\
\text { représentées par la moyen- } \\
\text { ne arithmétique des préci- } \\
\text { pitations recueillies dans } \\
12 \text { stations pendant la pé- } \\
\text { riode } 1^{\text {er novembre- } 1 \text { mars. }}\end{array}\right.$ \\
\hline $\begin{array}{c}\text { 1er mai-31 juillet et } \\
1^{* r} \text { mai-31 aoull : }\end{array}$ & $\left\{\begin{array}{l}\mathrm{P}_{\mathrm{H}}=\text { précipitations totales } \\
\text { représentées par la moyen- } \\
\text { ne arithméticue des préci- } \\
\text { pitations recueillies dans } \\
12 \text { slations pendant la pé- } \\
\text { riode } 1 \text { er novembre-30 avril. }\end{array}\right.$ \\
\hline $\begin{array}{l}\text { 1er juin-31 aoult et } \\
1^{\text {or juin-30 sept. : }}\end{array}$ & $\left\{\begin{array}{l}S=\text { Réserves naturelles (li- } \\
\text { quides ou solides) accu- } \\
\text { mulées dans le bassin } \\
\text { entre le 1er novembre e't } \\
\text { le } 31 \text { mai (représentées par } \\
\left.1,4 \mathrm{P}_{\mathrm{H}}-\mathrm{E}_{\mathrm{H}}\right) \text {. }\end{array}\right.$ \\
\hline 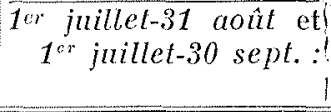 & $\begin{array}{l}\text { Ecoulement moyen du mois } \\
\text { de Juin. }\end{array}$ \\
\hline
\end{tabular}
et élement H choisi pour la corrélation avec l'écoulement 
Au début, nous avons utilisé les précipitations de la période d'hiver s'étendant du $1{ }^{\text {er novembre }}$ à la date de la prévision; par la suite, nous avons constaté qu’à partir du 1er juin, l'élément « réserves naturelles » était en meilleure corrélation avec l'écoulement à venir', et enfin, au $1^{\text {er }}$ juillet, nous avons utilisé la corrélation avec l'écoulement du mois de juin. Après le $1^{\text {er }}$ juillet, nous n'avons plus énoncé de prévisions car les études ne mettaient pas en évidence de corrélations satisfaisantes.

Les divers choix précédents n'ont pas été opérés dès le départ car nos études sont récentes et les calculs ont pratiquement été effectués au fur et à mesure des besoins immédiats résultant de notre objectif qui était de publier, au début de chaque mois, des prévisions immédiatement utilisables sur les perspectives offertes par la période subséquente allant jusqu'au $1^{\text {er }}$ septembre ou octobre.

C'est dire que les résultats acquis sont encore susceptibles d'amélioration et nous nous y employons en ce moment en cherchant de meilleures corrélations par un choix plus judicieux des éléments $\mathrm{H}$ et en améliorant les méthodes de mesure des diverses grandeurs hydrologiques et météorologiques considérées.

Nous allons examiner maintenant plus en détail le cas de la prévision faite au $1^{\text {er }}$ avril.

Le tableau II résume la suite des calculs effectués dans ce cas particulier. La valeur probable $\overline{\mathrm{E}}$ a été déplacée de $610 \mathrm{~mm}$ à $440 \mathrm{~mm}$ par la connaissance des précipitations d'hiver et l'étude de la corrélation. La zone de dispersion a été réduite d'un quart.

TABLEAU II

PIEEVISION DES APPORTS POUR LA PERIODE

$1^{\text {er }}$ AVRIL-31 AOUT 1949

\section{Drac all Sautet}

Résumé de la méthode á employer

et résultats des calculs

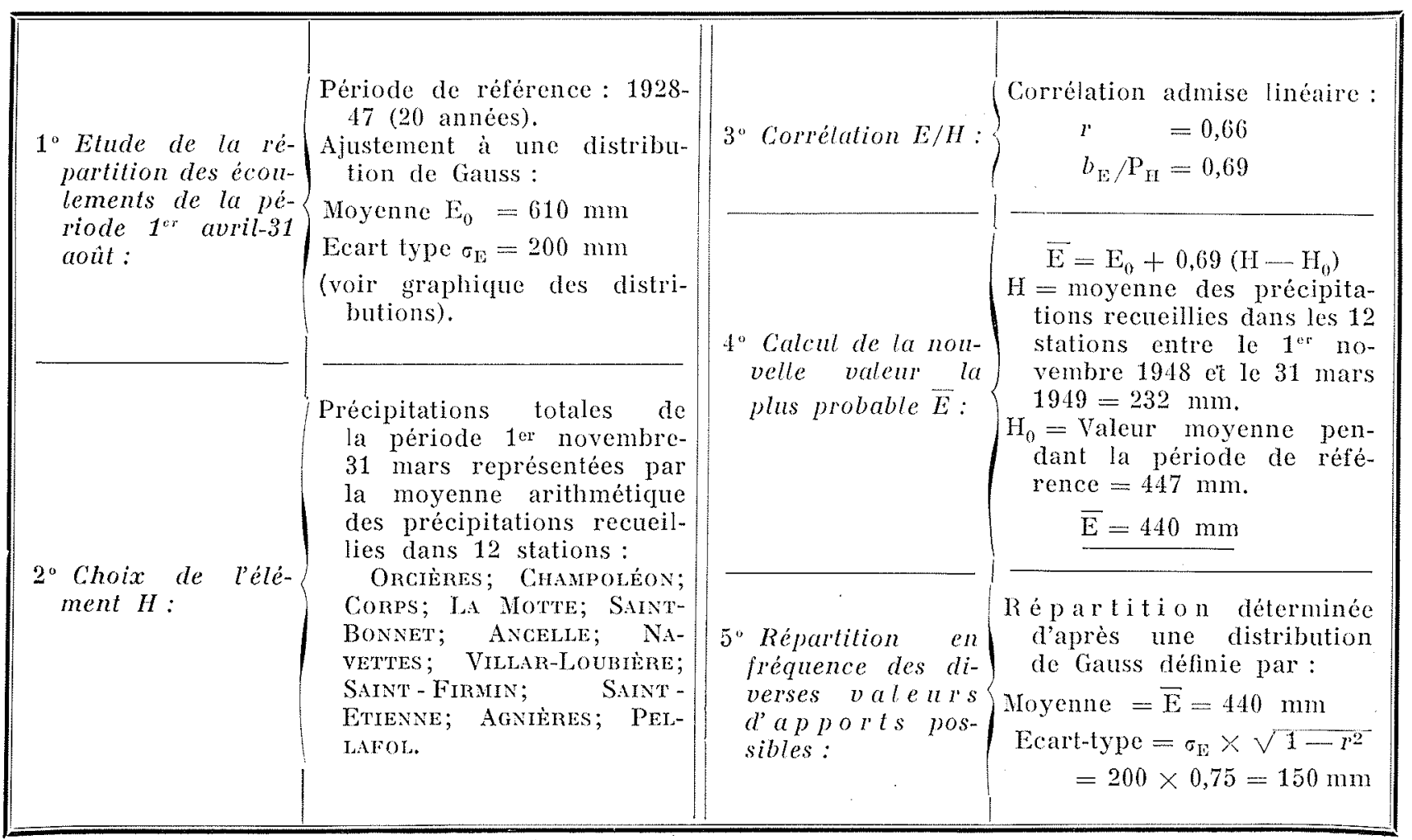


Le tableau (III) est la reproduction résumée du bulletin de prévisions correspondant tel qu'il a été diffusé par notre service aux autres services intéressés d'E.D.F. et en premier lieu celui des Mouvements d'Energie chargé de fixer les programmes de production des usines.

TABreaU IIII

PREVISION DES APPORTS POUR LA PÉRIODE $1^{\text {er }}$ AVRIL-31 AOUT 1949

\section{Drac all Santet}

Forme des bulletins de prévisions

$1^{\circ}$ Valeurs caractéristiques de l'écoulement pour

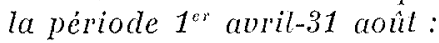

\begin{tabular}{|c|c|c|c|c|c|}
\hline \multirow[b]{3}{*}{$\begin{array}{l}\text { Valeur des apports } \\
\text { en mm ou en } M . m^{3}\end{array}$} & \multicolumn{5}{|c|}{$\begin{array}{l}\text { Nombre de fois sur cent } \\
\text { oit les apports indiqués sont } \\
\text { EN moYexne Dípassés }\end{array}$} \\
\hline & 95 & 90 & 50 & 10 & 5 \\
\hline & 280 & 350 & 610 & 870 & 940 \\
\hline
\end{tabular}

$2^{\circ}$ Probabilités d'apports pour la période $1^{\text {or }}$ avril91 aont résullant de l'observation des éléments mét'orologiques de l'hiver 1948-49:

\begin{tabular}{|c|c|c|c|c|c|}
\hline \multirow[b]{3}{*}{$\begin{array}{l}\text { Valeur des apports } \\
\text { en mm ou en W. m" }\end{array}$} & \multicolumn{5}{|c|}{$\begin{array}{l}\text { Probabilite de dépasser } \\
\text { les apports indiqués }\end{array}$} \\
\hline & $95 \%$ & $90 \%$ & $50 \%$ & $10 \%$ & $5 \%$ \\
\hline & 190 & 250 & 440 & 630 & 690 \\
\hline
\end{tabular}

$3^{\circ}$ Probabilités de remplissage de la réserve et des apports utilisables (capacité de la réserve $100 \mathrm{M}$. m: ou $100 \mathrm{~mm}$ ) :

\begin{tabular}{|c|c|c|c|}
\hline \multirow{2}{*}{$\begin{array}{c}\text { Taux de sécurité } \\
\text { en vue } \\
\text { du remplissage } \\
\text { de la réserve } \\
\text { au } 31 \text { août } 1949\end{array}$} & \multirow{2}{*}{$\begin{array}{c}\text { Situation } \\
\text { de la } \\
\text { réserve } \\
\text { ant } 1-1-49\end{array}$} & \multicolumn{2}{|c|}{$\begin{array}{c}\text { Apports utilisables } \\
\text { entre } \\
\text { le 1-4 et le } 31-8-49\end{array}$} \\
\hline & & en $\mathrm{mm}$ & \begin{tabular}{|} 
En nombre \\
d'heures \\
de marche \\
aut débit \\
maximum
\end{tabular} \\
\hline $90 \%$ & $20 \mathrm{~mm}$ & 170 & 530 heures \\
\hline $95 \%$ & & 110 & 350 hemres \\
\hline
\end{tabular}

La conclusion pratique à tirer des probabilités d'apports données au paragraphe 2" est indiquéc dans le paragraphe $3^{\circ}$. On y trouve les apports disponibles pour la production dans l'usine de pied de barrage suivant le taux de sécurité adopté pour le remplissage de la réserve à fin août $(95 \%$ ou $90 \%$ ). On n'y a pas considéré les taux de sécurité inférieurs à $90 \%$.

Je précise que ce bulletin a été établi à la date du 5 avril; c'est dire le court délai que nous nous autorisons pour rassembler les chiffres d'observations relatifs aux semaines précédentes et effectuer les diver's calculs. Pour obtenir ce résultat, nous avons été amenés à créer une organisation propre à E.D.F. comprenant des centres régionaux chargés de rassembler rapidement, de dépouiller et exploiter les données provenant des stations météorologiques et de mesure des débits utilisés.

La figure 1 représente graphiquement les résultats précédents et $\mathrm{y}$ ajoute la comparaison avec les apports effectivement observés. L'axe des abscisses correspond aux écoulements E exprimés en $\mathrm{mm}$ de hauteur de la lame d'eau équivalente sur toute la surface du bassin; les pourcentages indiqués en ordonnée correspondent à la fréquence ou à la probabilité des écoulements supérieurs à une valeur donnée $\mathbf{E}$. On trouve sur: ce graphique :

$1^{\circ}$ La distribution des ćcoulements $\mathrm{E}$ effectivement constatés pendant la série des années 19281947 pour la période $1^{\mathrm{cr}}$ avril-31 aont (graphique en escalier des fréquences cumulées);

2. La distribution de Gauss ajustée à la répartition réelle et définie par la moyenne $610 \mathrm{~mm}$ et l'écart-type $\sigma_{\mathrm{r}}=200 \mathrm{~mm}$;

$3^{\circ}$ La nouvelle distribution résultant de la corrélation entre $\mathrm{E}$ et $\mathrm{P}_{\mathrm{Ir}}$ et définie par la moyenne $440 \mathrm{~mm}$ (qui est la valeur que nous avons considérée comme la plus probable pour la période $1^{\mathrm{er}}$ avril-31 aout) et par l'écart-type $\sigma_{\mathrm{V}}^{\prime}=150 \mathrm{~mm}$.

Sur ce graphique est également tracée une quatrieme courbe qui représente la distribution tenant compte à la fois de la corrélation entre $E$ et $P_{I}$ et la corrélation entre $\mathbf{E}$ et les précipitations d'été $\mathrm{P}_{e}$.

A la fin de l'été nous avons en effet comparé les apports effectivement constatés au Sautet avec les probabilités que nous avions annoncées.

Pour la période $1^{\mathrm{er}}$ avril-31 août 1949, les apports ont été de $276 \mathrm{~mm}$ (représentés par la droite $V$ sur le dessin).

Ce nombre est inférieur à la plus faible valeur constatée depuis 1927 (288 $\mathrm{mm}$ en 1942). D'après 


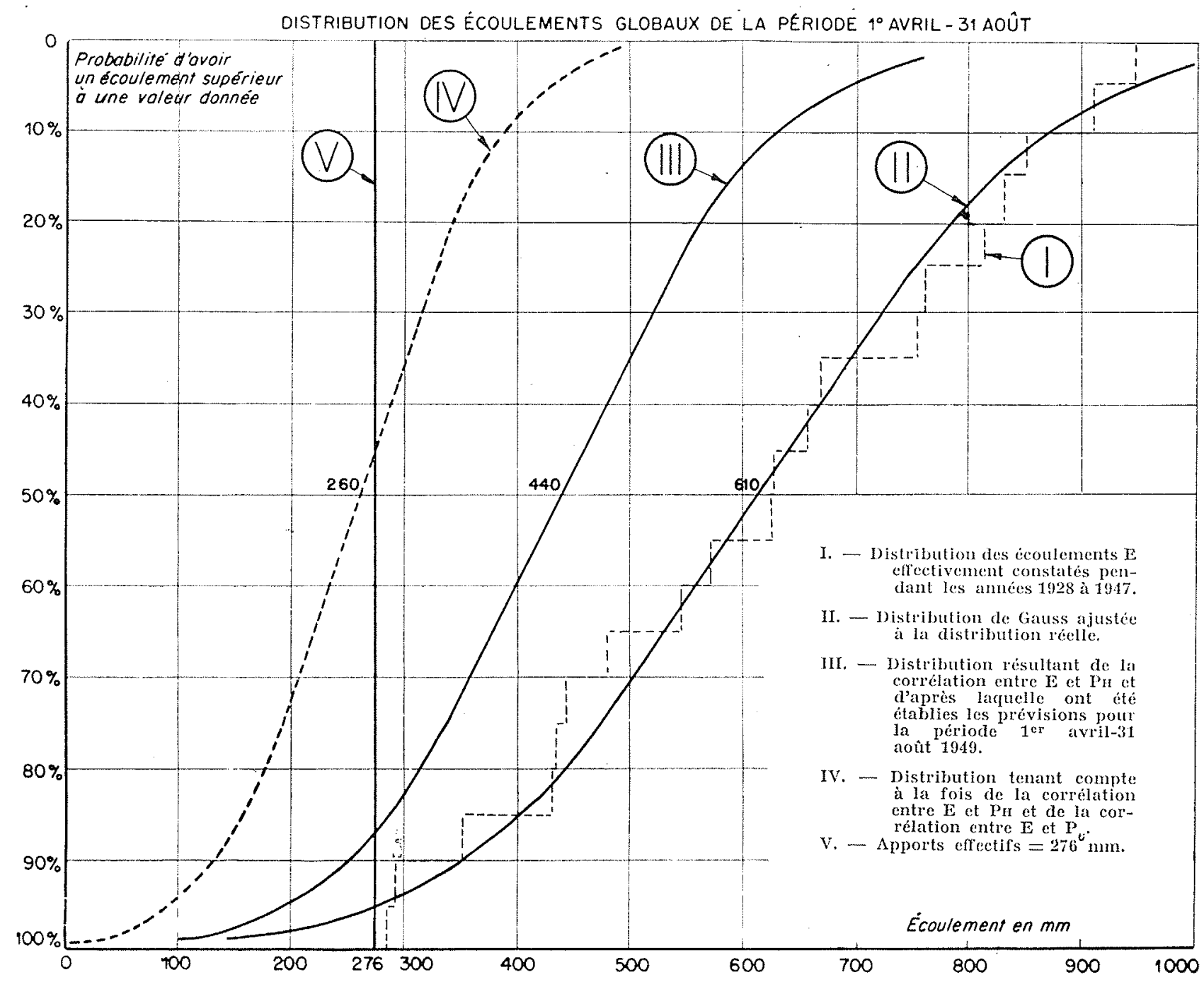

Fig. 1. - Pirévision des appontes 1er aviul-31 aout 1949.

Bassin : Drac au Sautet.

la distribution II, il y avait environ 95 chances sur 100 d'avoir des apports supérieurs à la valeur $276 \mathrm{~mm}$.

Si l'on considère la distribution III, base de nos prévisions, on constate que la valeur $276 \mathrm{~mm}$ correspond à la probabilité $88 \%$ d'avoir des apports supérieur's à cette valeur. Cette probabilité, bien qu'éloignée du taux $50 \%$ (correspondant à la valeur la plus probable), est néanmoins inférieure aux taux « de sécurité »- 95 et $90 \%$ - sur lesquels nous attirons l'attention des services utilisant les prévisions. En utilisant l'un ou l'autre de ces taux de sécurité, les prévisions effectuées peuvent done être considérées comme très satisfaisantes.

Néanmoins, il est intéressant de chercher à expliquer a posteriori l'écart relativement im- portant entre la valeur $276 \mathrm{~mm}$ et la valeur centrale de la distribution III, soit $440 \mathrm{~mm}$, par la considération de certains caractères de la période soumise à la prévision. Sans vouloir considérer tous les éléments susceptibles d'influencer les écoulements - éléments dont d'ailleurs certains sont inconnus et dont beaucoup ne sont malheureusement pas encore mesurés de façon systématique (ainsi l'intensité du rayonnement solaire, l'evaporation...) - nous avons cherché à nous faire une idée de l'influence des précipitations de la période $1^{\text {er }}$ avril31 août sur les écoulements. Plus précisément, nous avons calculé quelles auraient été nos prévisions si nous avions pu connaître à l'avance les précipitations de printemps et d'été.

Nous n'entrerons pas dans le détail du calcul qui a consisté à rechercher le lien de corrélation 
entre l'écart de l'écoulement à la valeur centrale de la prévision et les précipitations de la période. Nous avons trouvé que la faible valeur des précipitations du printemps et de l'été 1949, soit $300 \mathrm{~mm}$ (au lieu de 435 , moyenne constatée sur la série d'années 1928-1947), aurail eu pour effet de déplacer la valeur centrale de $450 \mathrm{~mm}$ à $260 \mathrm{~mm}$. Cette nouvelle valeur centrale est done très proche de la valeur réelle $276 \mathrm{~mm}$; la dispersion autour de cette valeur centrale est définie par un écart-type de $100 \mathrm{~mm}$.

L'écart entre la valeur observée et la valeur centrale de la prévision effectuće au début d'avril s'explique done entièrement par l'insuffisance des précipitations subséquentes.

On constate ainsi que, dans le cas du Drac, les précipitations (d'hiver et d'été) sont les eléments essentiels qui conditionnement l'écoulement de la période avril à août et qu'en particulier pour l'année 1949, le déficit important des précipitations des deux semestres suffit à rendre compte des apports extrêmement faibles et jamais constatés depuis 1922 qui ont été mesurés cet été à l'usine du Sautet (à titre de comparaison, on peut évaluer à $230 \mathrm{~mm}$ environ les apports de la même période en 1921).

\section{Romanche au Chambon.}

Le deuxième bassin sur lequel nous avons porté nos études est celui de la Romanche au Chambon.

Sans entrer dans le détail comme je viens de le faire pour le Drac, je dirai seulement que nous avons utilisé la même méthode, en considérant comme élément $H$, suivant les périodes, soit les précipitations totales d'hiver représen-

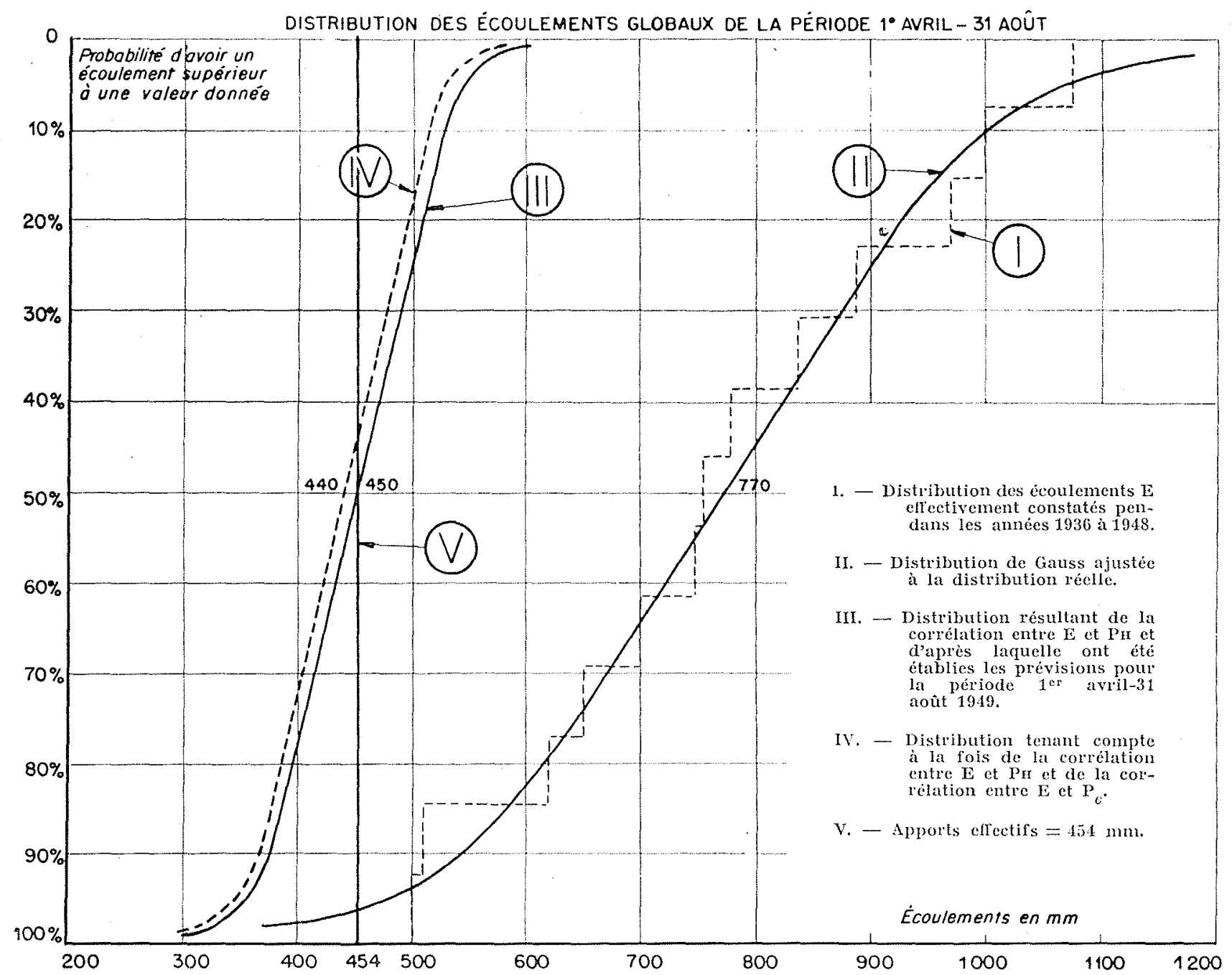

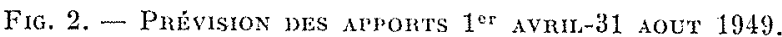

Bassin : Romanche au Chambon. 


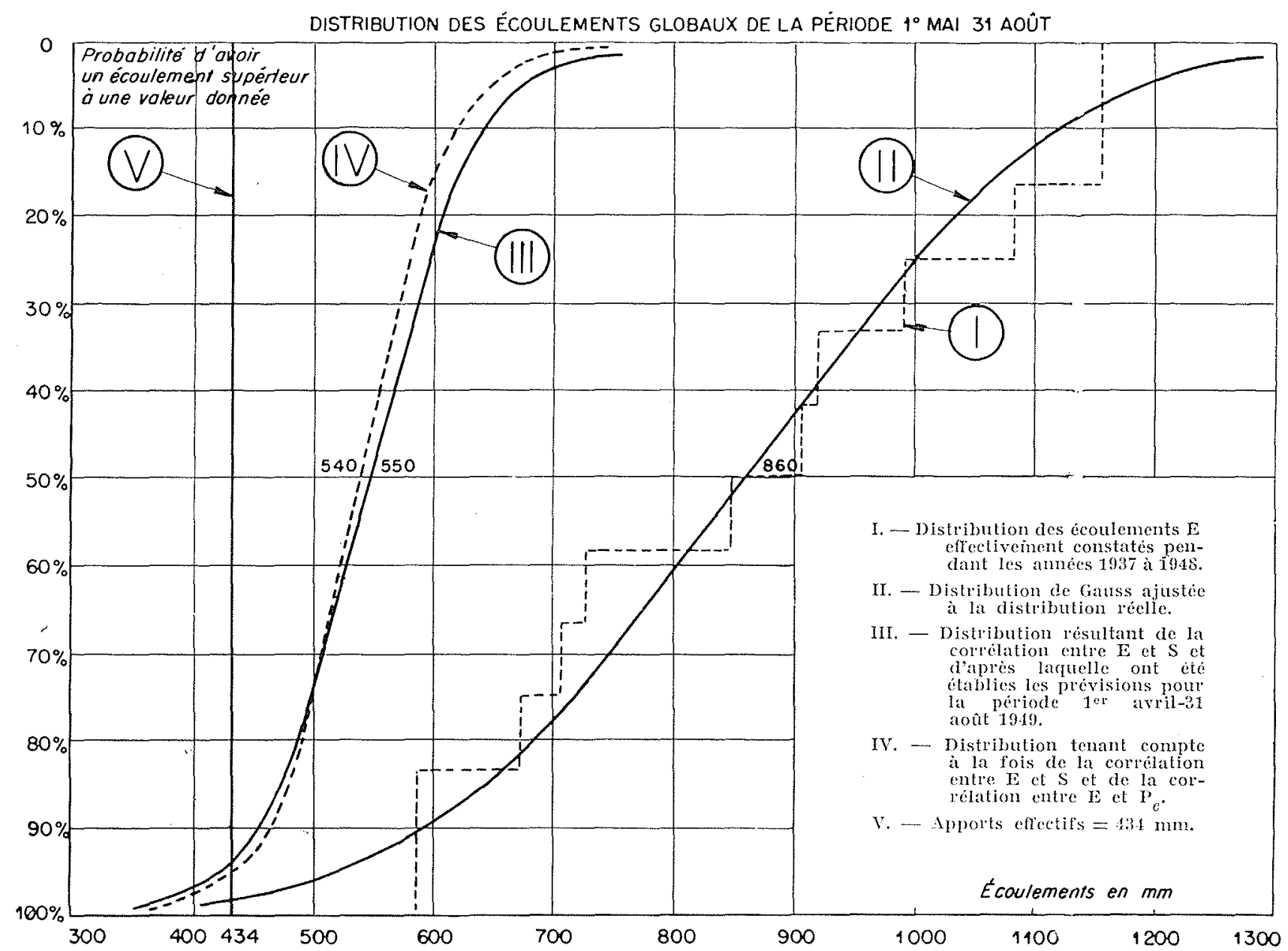

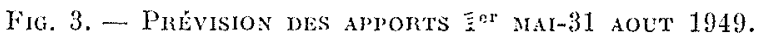
Bassin: Bissorte.

tées par la moyenne arithmétique de sept stations (Le Monetier, Besse, Villard-Notre-Dame, La Garde, Bourg d'O'isans, Omon, Verney d'Allemont), soit les « réserves naturelles», soit l'écoulement des mois précédents. Toutefois, je tiens à préciser que pour les premières prévisions, l'adoption des précipitations d'hiver comme élément $H$ n'a pas résulté d'un véritable choix, mais du fait que nous n'avons pas eu le temps de pousser nos calculs, étant tenus par la nécessité de publier rapidement des renseignements et parce que les corrélations que nous avions trouvées entre $E$ et $P_{\mathrm{Ir}}$ étaient déjà satisfaisantes $(x$ étant de l'ordre de 0,95 , donc nettement supérieur à ce que l'on trouvait pour le Drac, avec, il est vrai, seulement 13 années au lieu de 20 pour la série de référence).

Si nous considérons, maintenant, le cas des prévisions pour la période $1^{\text {er }}$ avril-31 août, les résultats sont condensés dans la figure 2 où sont représentés :
- La distribation empirique des écoulements de la séric d’année 1936-1948.

- La distribution de Gauss ajustée à la précédente :

$$
\begin{aligned}
& \text { Moyenne } \mathrm{E}_{0}=770 \mathrm{~mm} \\
& \text { Ecart-type } \sigma_{\mathrm{E}}=190 \mathrm{~mm}
\end{aligned}
$$

- La distribution résultant de la corrélation $\mathrm{E} / \mathrm{P}_{\mathrm{II}}$

Valeur la plus probable $\overline{\mathrm{E}}=450 \mathrm{~mm}$

Ecart-type $\sigma_{\mathrm{E}}^{\prime} \quad=60 \mathrm{~mm}$

(soit $30 \%$ seulement de $\sigma_{\mathrm{E}}$ ).

- La distribution résultant de l'étude de la corrélation avec les précipitations d'été (étude faite a posteriori pour discriminer la part d'influence de ces précipitations sur l'écoulement).

- L'écoulement effectivement constaté au barrage du Chambon pendant la période, soit $454 \mathrm{~mm}$. 
On constate d'après ces graphiques que la $v a-$ leur annoncée comme la plas probable pour $\mathrm{E}$ est trés sensiblement égale à la valeur conslaté: 450 contre $454 \mathrm{~mm}$, et qu'en outre elle est in/erieure à la valeur la plus faible de la série de référence de 12 ans, soit $500 \mathrm{~mm}$. Ce résultat fait ressortir de manière particulièrement frappante la valeur des informations prévisionnelles tirćes de l'étude des corrélations. La valeur de $375 \mathrm{~mm}$ ayant $90 \%$ de chance d'être dépassée suivant lá courbe III constitue ainsi indubitablement une base prudente pour la gestion du réservoir au cours de l'été.

On peut s'étonner de ce que la valeur constatéc s'écarte si peu de la valeur centrale prévue, malgré l'insuffisance des précipitations de la période avril-août qui n'a pu naturellement être prise en compte au moment de la prévision.

Mais l'étude de la corrélation entre les précipitations de la période avril-août et les écarts (écoulement réel - valeur centrale de la prévision) nous a montré que cette corrélation était très faible.

Sur la figure 2, les courbes III et IV sont très voisines l'une de l'autre. Dans le cas de la période $1^{\text {er }}$ mai-31 août, la corrélation envisagée étant, en fait, nulle, les deux courbes sont pratiquement confondues. Aînsi, si à la date du $1^{\text {er }}$ avril nous avions su que les précipitations des cing mois à venir allaient être au total :

- $250 \mathrm{~mm}$ au lieu de 350 (moyenne des années 1936 a 1948), soit 71 \% de la moyenne, nous aurions annoncé comme valeur la plus probable de $\mathbf{E}$ :

- $440 \mathrm{~mm}$, alors que notre prévision a réellement indiqué $450 \mathrm{~mm}$.

Ces divers résultats peuvent s'interpréter si l'on considère que le bassin de la Romanche ayant un caractère glaciaire plus accentué que celui du Drac, les écoulements d'été y sont conditionnés pour une grande part par la fusion des glaciers. Or celle-ci varie, d'une manière générale et en moyenne, en sens inverse des precipilations. Dans le hassin de la Romanche, il semble bien que ces deux actions inverses se compensent presque exactement.

$$
\because *
$$

L'exemple des deux bassins précédents - au sujet desquels je dois ajouter que les prévisions faites après le $1^{\text {er }}$ avril ont été également satisfaisantes, eu égard au caractère obligatoirement rapide et relativement sommaire de nos études, - constitue done pour nous un encouragement sérieux.
Les résultats n'ont pas été toujours aussi satisfaisants pour d'autres bassins que nous avons commencé à étudier.

\section{Bassin de Bissorte.}

La figure 3 donne, comme dans les deux exemples précédents, les diverses distributions envisagées at situc les apports constatés pour la période 1 cr mai-31 août.

$\mathrm{F}_{0}$ étant égal à $860 \mathrm{~mm}$, on constate que la corrélation $\mathrm{E} / \mathrm{H}$ a permis de rapprocher sensiblement la valeur la plus probable $(550 \mathrm{~mm})$ de la valeur effectivement constatée (434 mm). De plus, cette valeur la plus probable est, comme pour la Romanche, sensiblement inférieure à la valeur la plus faible constatée sur la période de référence de 12 ans ayant servi aux calculs, soit $588 \mathrm{~mm}$. L'écoulement constaté est sensiblement égal à la valeur annoncée comme correspondant à la limite inférieure des apports avec le taux de sécurité $95 \%$. Le taux de sécurité $90 \%$ se serait donc trouvé en défaut, mais avec une insuffisance de $30 \mathrm{~mm}$ seulement.

Par ailleurs, l'influence de la pluie d'été se traduit par une valeur centrale de $540 \mathrm{~mm}$ peu différente du chiffre correspondant de notre prévision et qui laisse subsister un écart encore important avec la valeur $434 \mathrm{~mm}$.

Cet écart, considéré isolément, n'a rien d'invaraisemblable, surtout si l'on tient comple de l'erreur d'estimation sur les paramètres statistiques calculés à partir d'un échantillon de douze années seulement. Nćanmoins, nous devons le rapprocher du fait que nous trouvons des écarts supérieurs encore pour les périodes subséquentes afférentes au mème bassin et pour quelques autres bassins alpestres ou pyrénéens. Il nous parait peu satisfaisant d'attribuer tous ces écarts à l'erreur d'échantillonnage combinée avec un hasard malheureux. Peut-être y a-t-il lieu d'incriminer un phénomine physique perturbateur. 'Tous les bassins en question sont des bassins de petite superficie dont l'altitude minimum est voisine de $2.000 \mathrm{~m}$ et qui possédaient soit de petits glaciers (Bissorte), soit des névés susceptibles, peut-être, d'exercer une action régulatrice non négligeable sur les débits de l'été. Or, ces réserves solides ont été très entamćes, sinon entièrement consommées, au cour's de ces dernières années. Lors de la sécheresse exceptionnelle de cette saison, on n'aurait donc pas bénéficié de la compensation résultant d'une fusion plus intense de ces réserves.

Ce n'est encore là qu'une simple hypothèse sur 
laquelle nous ne pouvons pas actuellement nous prononcer.

$$
\text { * }
$$

Pour terminer cette rapide présentation de nos travaux et des premiers résultats acquis, je tiens a bien marquer le caractère essentiellement pratique du but à atteindre : renseigner le plus rapidement possible les services intéressés d'E.D.F. sur les apports à attendre dans les usines et en premier lieu les réservoirs. Ceci nous impose d'aller vite et de rechercher des méthodes simples d'exploitation des renseignements qui sont fournis par les stations d'observation.

Je ne disconviens pas que cette facon de faire peut nuire parfois à la rigueur statistique (en particulier lorsqu'il s'agit d'ajuster une distribution de Gauss à une distribution empirique d'écoulements). Nous nous efforcons actuellement de perfectionner la méthode, tout en l'étendant à d'autres cas, dont certains de nature différente.

\section{I S C U S S I O N}

Tout en reconnaissant la validité de l'explication donnce par M. Fenry a fait que les points effectivement observes s'écartent de façon un peu anormale de la mojenne de la distribution calculée, M. Monfat demande s'il n'y a pas lieu d'cnvisager d'abord une autre interprétation : la distribution réclle de la prévision ne risque-t-clle pas d'ètre systématiquement différente de la distribution calculée (en particulier la dispersion serait sous-estimée) du fait qu'on a peutêtre sélectionné, pour effectuer la prévision, les données pluviométriques et la période qui fournissaient la meilleure corrélation?

M. Fenry répond à celà que, sans nier les possibilités de cette cause de surévaluation systématique des cocficients de corrélation, il croit qu'elle a été réduite a des proportions très modestes pour trois raisons. La premiere e'est que les paramètres adoptés ne sont pas brutalement, dans chaque cas particulier, ceux qui donnent la corrélation numérique maximum; ils résultent d'un jugement d'ensemble où la valeul' obtenue pour la corrélation n'est qu'un élément et où les considérations physiques dominent; la seconde c'est que les indications données par ce dernier élément étaient assez concordantes, dans l'ensemble; la troisième c'est que les valeurs des coefficients de corrélation et les formules de régression trouvées variaient en général assez peu quels que soient les parametres et elements de base adoptes, dans la zone pratique d'incertilude oit l'on pouvat choisir ces èlements.

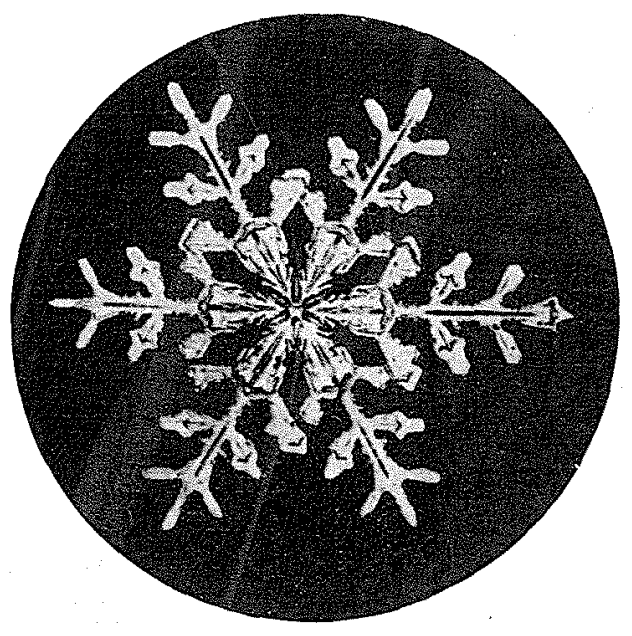

